\title{
DYNAMIC CHARACTERISTICS AND STABILITY OF CYLINDRICAL TEXTURED JOURNAL BEARING
}

\author{
T S Reddy Ganji $1^{1}$ and S K Kakoty ${ }^{2}$ \\ ${ }^{1}$ Department of Mechanical Engineering, Indian Institute of Technology Guwahati, \\ Assam, India \\ t.ganjieitit.ernet.in \\ ${ }^{2}$ Department of Mechanical Engineering, Indian Institute of Technology Guwahati, \\ Assam, India \\ sashinditg.ernet.in
}

\begin{abstract}
The dynamic characteristics of cylindrical textured hydrodynamic journal bearing are presented in this paper. The Reynolds equation is solved numerically in a finite difference grid in an iterative scheme satisfying the appropriate boundary conditions. Stiffness and damping coefficients of fluid film and stability parameter are found using the first-order perturbation method for different eccentricity ratios and various texture parameters like texture depth and texture density.. From the present analysis, it has been found that stability is enhanced with increase in texture depth, whereas there is an optimum texture density corresponding to the maximum stability of the bearing.
\end{abstract}

\section{KEYWORDS}

Cylindrical Texture, Stiffness and Damping coefficients, Hydro Dynamic Lubrication, Journal Bearing

\section{NOMENCLATURE}

$C$ Radial clearance (m)

$D$ Diameter of the bearing $(\mathrm{m})$

$D_{r r}, D_{\phi \phi}, D_{r \phi}, D_{\phi r}$ Damping coefficients (Ns/m)

$\bar{D}_{r r}, \bar{D}_{\phi \phi}, \bar{D}_{r \phi}, \bar{D}_{\phi r}$ Non-dimensional damping coefficients, $\bar{D}_{i j}=D_{i j} C^{3} / \mu R^{3} L$

$e, \varepsilon$ Eccentricity, $\varepsilon=e / C$

$e_{0}, \varepsilon_{0}$ Steady-state eccentricity, $\varepsilon_{0}=e_{0} / C$

$e_{1}, \varepsilon_{1}$ Perturbed eccentricity, $\varepsilon_{1}=e_{1} / C$

$h, \bar{h}$ Film thickness, $\bar{h}=h / C$

$\Delta h$ Variation of film thickness due to the presence of the texture (m)

$\overline{\Delta h}$ Dimensionless Variation of film thickness due to the presence of the texture, $\overline{\Delta h}=\Delta h / C$

$L$ Bearing length

DOI : 10.14810/ijmech.2014.3301 
$M, \bar{M}$ Mass parameter, $\bar{M}=M C \omega^{2} / W_{0}$

$p, \bar{p}$ Lubricant Pressure, $\bar{p}=p C^{2} / 6 \eta U R$

$\bar{p}_{0}, \bar{p}_{1}, \bar{p}_{2}$ Steady-state and perturbed dimensionless pressures

$R$ Journal radius $(\mathrm{m})$

$r_{p}$ Base radius of dimple

$r_{1}$ Half-length of imaginary textured square cell

$r_{x}, r_{y}, r_{z}$ Texture dimensions along $\mathrm{x}, \mathrm{y}$ and $\mathrm{z}$ directions

$S$ Sommerfeld number, $S=(\eta N / p) \times(R / C)^{2}$

$S_{p}$ Texture area density, $S_{p}=\pi r_{p}^{2} / 4 r_{1}^{2}$

$U$ Linear velocity $(\mathrm{m} / \mathrm{s})$

$W$ Load carrying capacity $(\mathrm{N})$

$\bar{W}$ Dimensionless Load carrying capacity, $\bar{W}=W C^{2} / 6 \eta U R^{2} L$

$x, z$ Cartesian coordinates

$\bar{x}, \bar{z}$ Dimensionless Cartesian coordinates

$x_{1}, z_{1}$ Local coordinates with their origin at the center of a single dimple cell

$\overline{x_{1}}, \overline{z_{1}}$ Local dimensionless coordinates with their origin at the center of a single dimple cell

$\alpha$ Textured portion in circumferential direction

$\beta$ Textured portion in axial direction

$\phi$ Attitude angle

$\eta$ Dynamic viscosity (Pas)

$\mu$ Coefficient of friction

$\bar{\mu}$ Friction variable, $\bar{\mu}=\mu(R / C)$

$\theta$ Angular coordinate

$t$ Time (s)

$\omega, \omega_{p}$ Journal rotational speed $(\mathrm{rad} / \mathrm{s})$, frequency of journal vibration

$\tau$ Non dimensional time, $\tau=\omega_{p} t$

$\lambda$ Whirl ratio, $\lambda=\omega_{p} / \omega$

$\Lambda$ Bearing number, $\Lambda=6 \eta \omega / p(C / R)^{2}$

( ) Steady state value

\section{Subscripts}

max : Maximum value

min : Minimum value 


\section{INTRODUCTION}

Micro dimples are developed by incremental stamping using the structured tool. The structured tool is manufactured by focused ion beam sputtering. Interference lithography is also used for producing micro dimples on surfaces. Matsumura et al. [1] have studied and developed micro fabrication techniques on cylinder surface. Matsumura et al. [2] have also developed some micro dimples on aluminum plates. Tala Ighil et al. [3] presented an analysis of cylindrical textured bearing and showed that the performance of bearing is influenced by textured surface.The dynamic characteristics of hydrodynamic journal bearings lubricated with micro polar fluids are presented by Das et al. [4].The dynamic behavior of axial grooved journal bearings in terms of stiffness and damping coefficients of fluid film and stability are found by Roy and Laha [5].Brizmer and Kligerman have found that both load capacity and attitude angle of the journal bearings could be improved by using partial LST mode at low eccentricities [6].Li and Wang have investigated the influence of the radius of the dimples on the tribological performance of a journal bearing. Results showed that the friction coefficient increased with both the width and the height of bulges in the case of journal bearing under light and moderate loading conditions [7]. A linearized perturbation approach has been used by Pai et al. [8] to study the stability characteristics of tri-taper journal using the Reynolds boundary condition. Dynamic characteristics in terms of stiffness, damping coefficients, the critical mass and whirl ratio are determined for various values of size of material characteristic length and the coupling number for a four-lobe journal bearing lubricated with a micro polar fluid by Chetti[9]. The stability characteristics of water lubricated journal bearings having three axial grooves are obtained theoretically by Vijaya Kini et al. [10].

The present work aims to find the dynamic characteristics of positive cylindrical textured journal bearing, i.e. the effect of texturing on dynamic characteristics like Mass parameter, Whirl ratio, Stiffness and Damping coefficients.

\section{NUMERICAL FORMULATION}

The Reynolds equation in non-dimensional form for an incompressible fluid can be written as

$$
\frac{\partial}{\partial \theta}\left(\bar{h}^{3} \frac{\partial \bar{p}}{\partial \theta}\right)+\left(\frac{D}{L}\right)^{2}\left(\bar{h}^{3} \frac{\partial^{2} \bar{p}}{\partial \bar{z}^{2}}\right)=\frac{\partial \bar{h}}{\partial \theta}+2 \lambda \frac{\partial \bar{h}}{\partial \tau}
$$

Where,

$$
\theta=\frac{x}{R}, \quad \bar{z}=\frac{z}{L / 2}, \quad \bar{h}=\frac{h}{C}, \quad \bar{p}=\frac{p C^{2}}{6 \eta U R}, \quad \tau=\omega_{p} t, \quad \lambda=\frac{\omega_{p}}{\omega}
$$

The film thickness for textured journal bearing, $h$, can be written as follows:

$$
h_{0}=h_{\text {smooth }}(\theta)-\Delta h(\theta, z)
$$




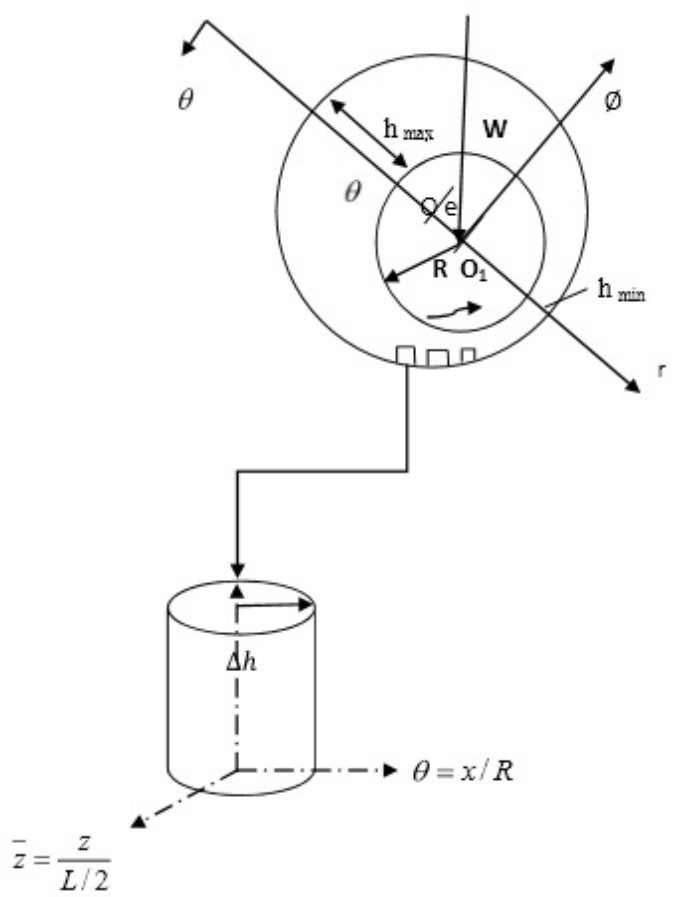

Figure 1: Cylindrical Textured Journal Bearing

Non dimensional film thickness can be written as

$$
\begin{array}{ll}
\overline{h_{0}}=1+\varepsilon_{0} \cos \theta-\overline{\Delta h} & \text { if }{\overline{x_{1}}}^{2}+{\overline{z_{1}}}^{2} \leq 1 \\
\overline{h_{0}}=1+\varepsilon_{0} \cos \theta & \text { if }{\overline{x_{1}}}^{2}+{\overline{z_{1}}}^{2}>1
\end{array}
$$

The pressure and film thickness can be expressed for small amplitude of vibration as

$$
\begin{aligned}
& \bar{p}=\bar{p}_{0}+\varepsilon_{1} e^{i \tau} \bar{p}_{1}+\varepsilon_{0} \phi_{1} e^{i \tau} \bar{p}_{2} \\
& \bar{h}=\bar{h}_{0}+\varepsilon_{1} e^{i \tau} \cos \theta+\varepsilon_{0} \phi_{1} e^{i \tau} \sin \theta
\end{aligned}
$$

The following three equations are obtained by substituting equation (5) into equation (1) and retaining the first order terms and equating the coefficients of $\varepsilon_{0}, \varepsilon_{1} e^{i \tau}$ and $\varepsilon_{0} \phi_{1} e^{i \tau}$

$$
\frac{\partial}{\partial \theta}\left(\bar{h}_{0}^{3} \frac{\partial \overline{p_{0}}}{\partial \theta}\right)+\left(\frac{D}{L}\right)^{2} \frac{\partial}{\partial \bar{z}}\left(\bar{h}_{0}^{3} \frac{\partial \overline{p_{0}}}{\partial \bar{z}}\right)=\Lambda \frac{\partial \overline{h_{0}}}{\partial \theta}
$$




$$
\begin{aligned}
& \frac{\partial}{\partial \theta}\left(\bar{h}_{0}^{3} \frac{\partial \overline{p_{1}}}{\partial \theta}\right)+\left(\frac{D}{L}\right)^{2} \frac{\partial}{\partial \bar{z}}\left(\bar{h}_{0}^{3} \frac{\partial \overline{p_{1}}}{\partial \bar{z}}\right)+3 \frac{\partial}{\partial \theta}\left({\overline{h_{0}}}^{2} \frac{\partial \overline{p_{0}}}{\partial \theta} \cos \theta\right) \\
& +\left(\frac{D}{L}\right)^{2} \frac{\partial}{\partial \bar{z}}\left(\bar{h}_{0}^{2} \frac{\partial \overline{p_{0}}}{\partial \bar{z}} \cos \theta\right)=-\Lambda \sin \theta+i 2 \Lambda \lambda \cos \theta \\
& \frac{\partial}{\partial \theta}\left(\bar{h}_{0}^{3} \frac{\partial \overline{p_{2}}}{\partial \theta}\right)+\left(\frac{D}{L}\right)^{2} \frac{\partial}{\partial \bar{z}}\left(\bar{h}_{0}^{3} \frac{\partial \overline{p_{2}}}{\partial \bar{z}}\right)+3 \frac{\partial}{\partial \theta}\left(\bar{h}_{0}^{2} \frac{\partial \overline{p_{0}}}{\partial \theta} \sin \theta\right) \\
& +\left(\frac{D}{L}\right)^{2} \frac{\partial}{\partial \bar{z}}\left(\bar{h}_{0}^{2} \frac{\partial \overline{p_{0}}}{\partial \bar{z}} \sin \theta\right)=\Lambda \cos \theta+i 2 \Lambda \lambda \sin \theta
\end{aligned}
$$

Boundary conditions used for steady state and dynamic pressures are

$$
\overline{p_{i}}(\theta, 0)=\bar{p}_{i}(\theta, L)=0 \bar{p}_{i}(\theta, \bar{z})=\overline{p_{i}}(2 \pi, \bar{z}) \quad \text { Where } \overline{p_{i}}=\overline{p_{0}}, \overline{p_{1}}, \overline{p_{2}}
$$

The boundary conditions shown above should be complemented by the conditions at the boundaries of possible cavitation regions associated with each individual dimple. The Reynolds boundary condition (also known as the Swift-Stieber boundary condition) implies that the pressure gradient with respect to the direction normal to the boundary of the cavitation zone is zero and the dimensionless pressure inside the cavitation zone is also zero. Using an iterative solution scheme, it is simple to apply this condition to the Equations (6,7 and 8). Negative, i.e., sub-ambient pressures are changed to zero in each iterative cycle; the process converges, by numerical diffusion, to the required Reynolds condition [11].

Figure 1 shows the schematic representation of textured journal bearing. The texture shape used in this analysis is cylindrical. The texture distribution is uniform as shown in Fig. 2. When $r_{p}$ represents the radius of cylindrical dimple, $2 r_{1}$ is the length of the imaginary square cell as shown in the Fig. 2. Equations (6, 7 and 8) are solved numerically in a finite difference grid. GaussSiedel method with over relaxation has been used for solving the discretized Reynolds equations satisfying the boundary conditions. 


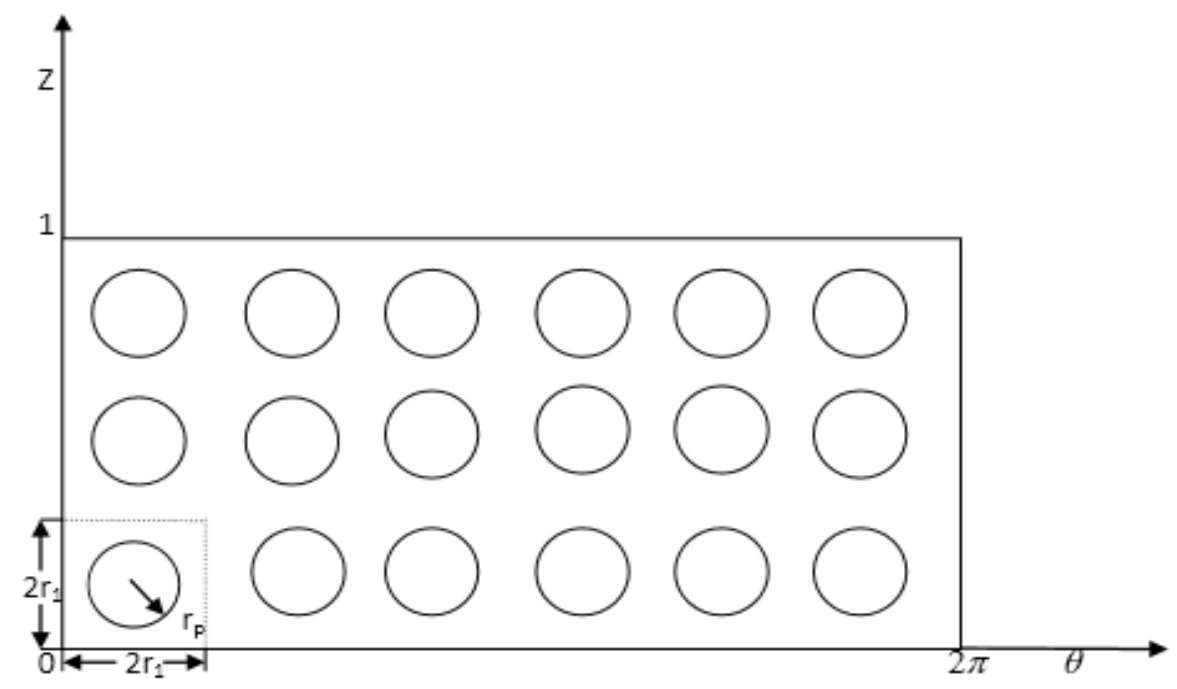

Figure 2: Distribution of textures on the bearing surface

As the dimples are very small, it has been observed that finite difference grid needs to be very fine for convergence. A grid size of $350 \times 50$ in the present work has been found to give gridindependent pressure distribution. The components of steady-state load carrying capacity are estimated by numerical integration of pressure as shown in equations ( 9 and 10). Steady state load and attitude angles are estimated as shown in equation (11 and 12).

$$
\begin{aligned}
& \bar{W}_{r}=-\int_{0}^{1} \int_{0}^{2 \pi} \bar{p}_{0} \cos \theta d \theta d \bar{z} \\
& \bar{W}_{t}=\int_{0}^{1} \int_{0}^{2 \pi} \bar{p}_{0} \sin \theta d \theta d \bar{z} \\
& \bar{W}_{0}=\sqrt{\bar{W}_{r}^{2}+\bar{W}_{t}^{2}} \\
& \phi_{0}=\tan ^{-1}\left(\frac{\bar{W}_{t}}{\bar{W}_{r}}\right)
\end{aligned}
$$

The expression for friction variable can be written as [12]

$$
\bar{\mu}=\mu\left(\frac{R}{C}\right)=\frac{\int_{0}^{2 \pi}(3 \bar{h}(\partial \bar{p} / \partial \theta)+1 / \bar{h}) d \theta}{6 \bar{W}}
$$


The stiffness and damping coefficients are given by

$$
\begin{aligned}
& \bar{K}_{r r}=-\operatorname{Re}\left(\int_{0}^{1} \int_{0}^{2 \pi} \bar{p}_{1} \cos \theta d \theta d \bar{z}\right) \\
& \bar{K}_{\phi r}=-\operatorname{Re}\left(\int_{0}^{1} \int_{0}^{2 \pi} \bar{p}_{1} \sin \theta d \theta d \bar{z}\right) \\
& \bar{D}_{r r}=-\operatorname{Im} \frac{\left(\int_{0}^{1} \int_{0}^{2 \pi} \bar{p}_{1} \cos \theta d \theta d \bar{z}\right)}{\lambda} \\
& \bar{D}_{\phi r}=-\operatorname{Im} \frac{\left(\int_{0}^{1} \int_{0}^{2 \pi-\bar{p}_{1}} \sin \theta d \theta d \bar{z}\right)}{\lambda} \\
& \bar{K}_{\phi \phi}=-\operatorname{Re}\left(\int_{0}^{1} \int_{0}^{2 \pi} \bar{p}_{2} \sin \theta d \theta d \bar{z}\right) \\
& \bar{K}_{r \phi}=-\operatorname{Re}\left(\int_{0}^{1} \int_{0}^{2 \pi} \bar{p}_{2} \cos \theta d \theta d \bar{z}\right) \\
& \bar{D}_{\phi \phi}=-\operatorname{Im} \frac{\left(\int_{0}^{1} \int_{0}^{2 \pi-\bar{p}_{2}} \sin \theta d \theta d \bar{z}\right)}{\lambda} \\
& \bar{D}_{r \phi}=-\operatorname{Im} \frac{\left(\int_{0}^{1} \int_{0}^{2 \pi} \bar{p}_{2} \cos \theta d \theta d \bar{z}\right)}{\lambda}
\end{aligned}
$$

Stiffness and damping coefficients are used in the equations of motion to obtain the following equations for getting non-dimensional mass parameter:

$$
\begin{aligned}
& \bar{M}=\frac{1}{\lambda^{2}\left(\bar{D}_{\phi \phi}+\bar{D}_{r r}\right)}\left[\begin{array}{c}
\left(\bar{K}_{r r} \bar{D}_{\phi \phi}+\bar{D}_{r r} \bar{K}_{\phi \phi}\right)-\left(\bar{K}_{\phi r} \bar{D}_{r \phi}+\bar{D}_{\phi r} \bar{K}_{r \phi}\right) \\
+\frac{\bar{W}}{\mathcal{E}_{0}}\left(\bar{D}_{r r} \cos \phi_{0}-\bar{D}_{\phi r} \sin \phi_{0}\right)
\end{array}\right] \\
& \bar{M}^{2} \lambda^{4}-\lambda^{2}\left[\bar{M}\left(\frac{\bar{W} \cos \phi_{0}}{\varepsilon_{0}}+\bar{K}_{\phi \phi}+\bar{K}_{r r}\right)+\left(\bar{D}_{r r} \bar{D}_{\phi \phi}-\bar{D}_{\phi r} \bar{D}_{r \phi}\right)\right] \\
& +\left(\bar{K}_{r r} \bar{K}_{\phi \phi}-\bar{K}_{\phi r} \bar{K}_{r \phi}\right)+\frac{\bar{W}}{\varepsilon_{0}}\left(\bar{K}_{r r} \cos \phi_{0}-\bar{K}_{\phi r} \sin \phi_{0}\right)=0
\end{aligned}
$$

Equations (15) and (16) are linear algebraic equations in $\bar{M}$ and $\lambda$. Solution of these will give $\bar{M}$ and $\lambda$. 


\section{RESULTS AND DISCUSSIONS}

\subsection{Characteristics of journal bearing}

The dynamic characteristics are presented in this section. Direct and cross stiffness coefficients for increasing texture depth are presented in Fig. 3, when direct and cross damping coefficients are presented in Fig. 4. The stability margin and whirl ratio have been presented in Fig. 5. These results are for $L / D=1, \varepsilon=0.6, S_{p}=0.8, \alpha=1, \beta=1$. It may be observed from Fig. 3 that the non-dimensional direct stiffness coefficient, $\bar{K}_{\phi \phi}$ increases with increase in texture depth and $\bar{K}_{r r}, \bar{K}_{r \phi}$ are decreases slightly with increase in texture depth. The non-dimensional cross stiffness coefficient, $\bar{K}_{\phi r}$ increases slightly with increase in texture depth. Further it has been observed from Fig. 4 that the non-dimensional direct damping coefficient, $\bar{D}_{\phi \phi}$ increases with increase in texture depth and $\bar{D}_{r r}$, decreases slightly with increase in texture depth. The nondimensional cross damping coefficients $\bar{D}_{\phi r}$ and $\bar{D}_{r \phi}$ decrease slightly with increase in texture depth.

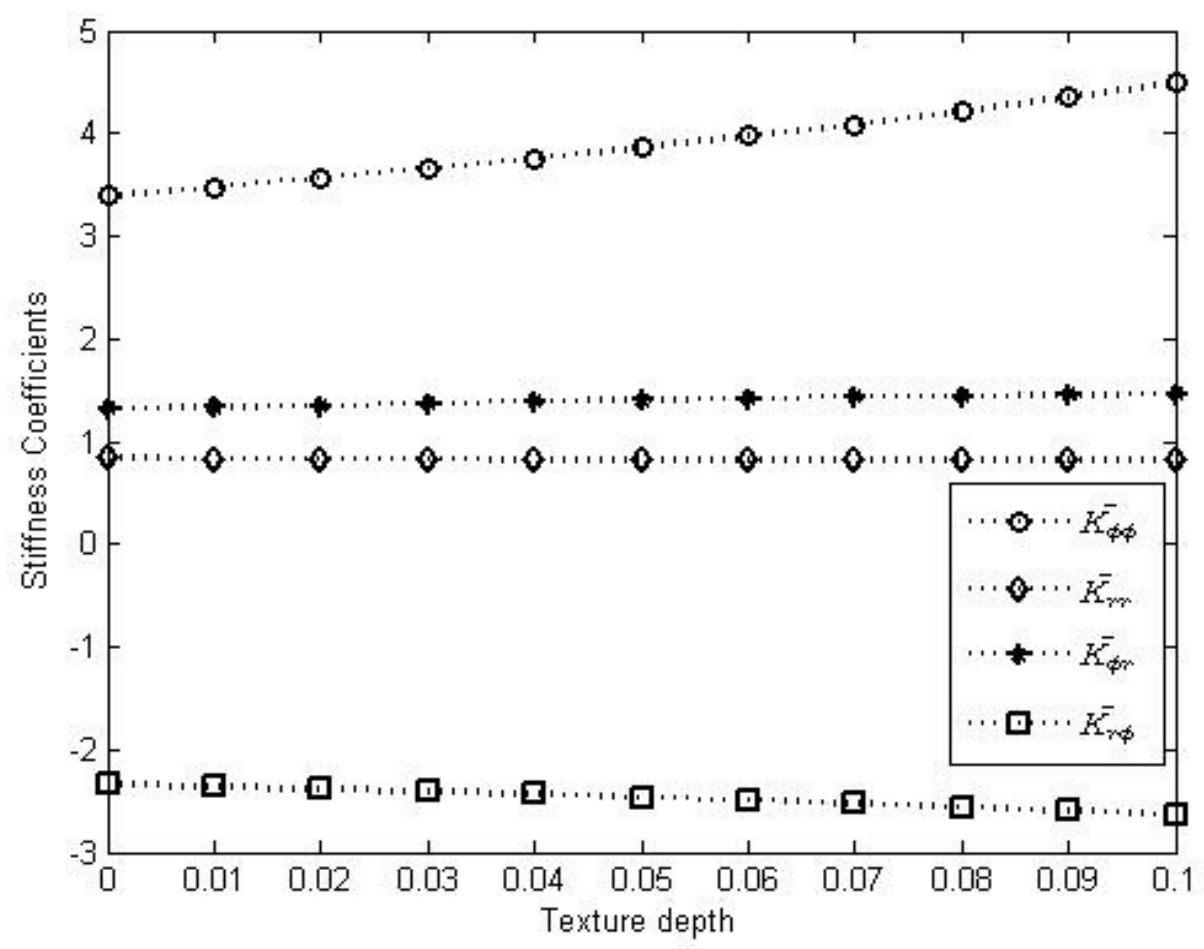

Figure 3:Stiffness coefficients vs. Texture $\operatorname{depth}\left(L / D=1, \mathcal{E}=0.6, S_{p}=0.8, \alpha=1, \beta=1\right)$ 
International Journal of Recent advances in Mechanical Engineering (IJMECH) Vol.3, No.3, August 2014

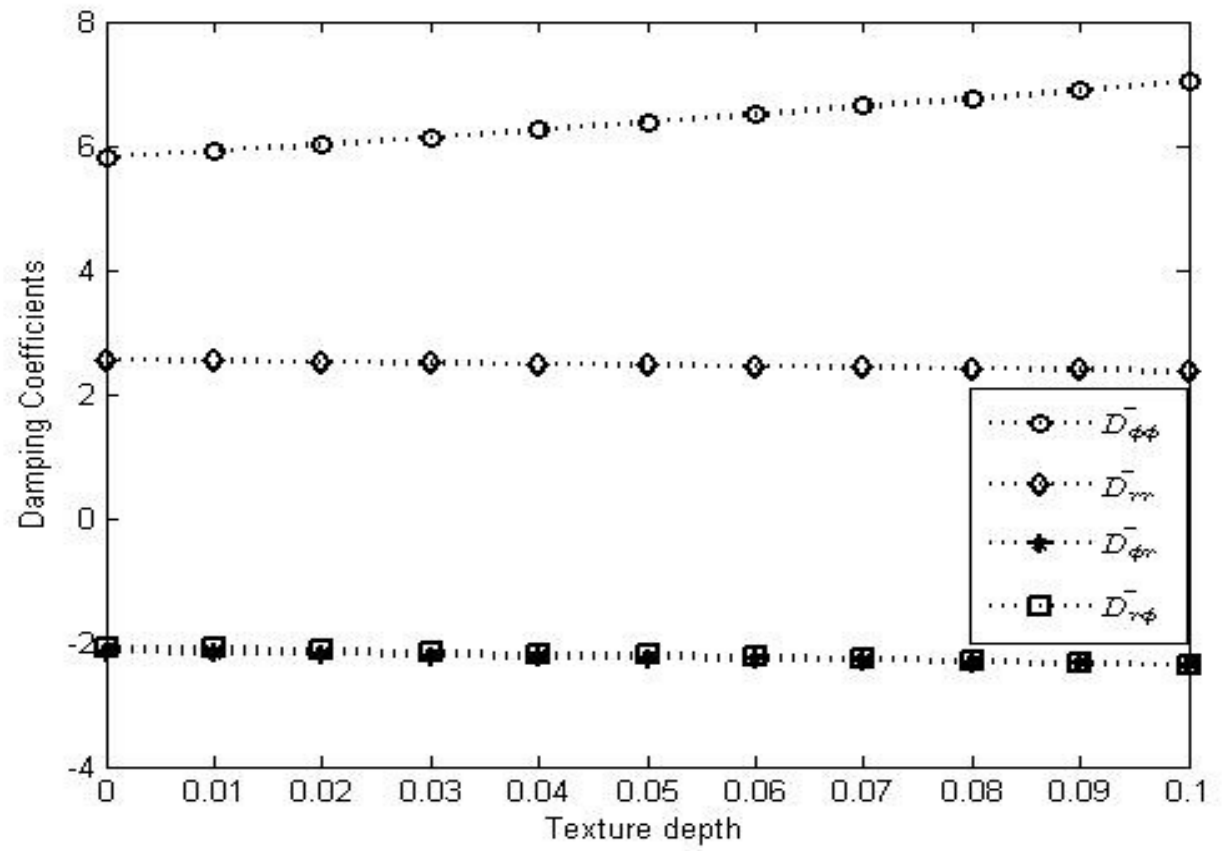

Figure 4:Damping coefficients vs. Texture $\operatorname{depth}\left(L / D=1, \varepsilon=0.6, S_{p}=0.8, \alpha=1, \beta=1\right)$

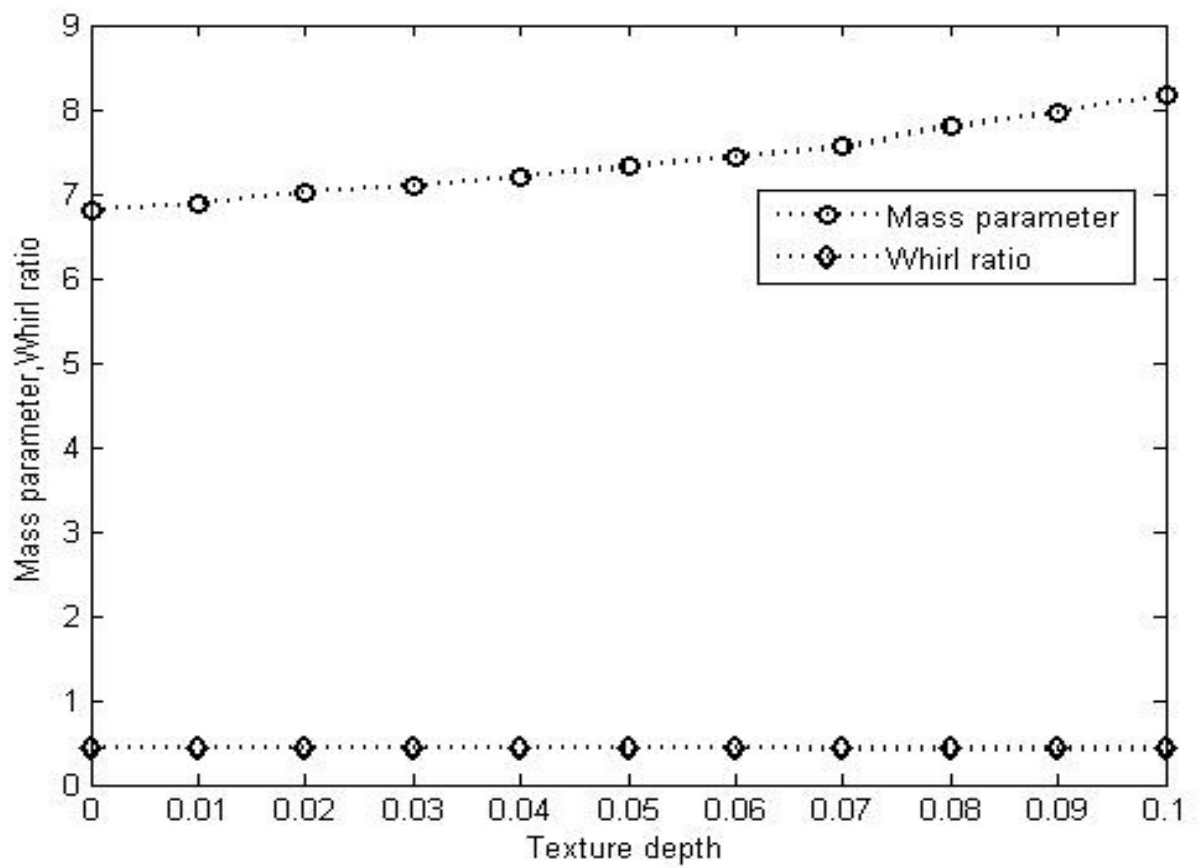

Figure 5: Mass parameter and whirl ratio vs. Texture $\operatorname{depth}\left(L / D=1, \mathcal{E}=0.6, S_{p}=0.8, \alpha=1, \beta=1\right)$ 
Critical mass parameter, a function of speedwhich is the measure of stability threshold, increases with increase in texture depth and whirl ratio decreases slightly with increase in texture depth as seen from Fig. 5.

Figure 6represents the variation of non-dimensional stiffness coefficients with eccentricity ratio for $L / D=1, S_{p}=0.8, \Delta \bar{h}=0.1, \alpha=1, \beta=1$. The non-dimensional direct stiffness coefficients, $\bar{K}_{\phi \phi}$ and $\bar{K}_{r r}$ increase slightly with increase in eccentricity ratio. The non-dimensional cross stiffness coefficient, $\bar{K}_{\phi r}$ decreases with increase in eccentricity ratio and $\bar{K}_{r \phi}$ increases with increase in eccentricity ratio.

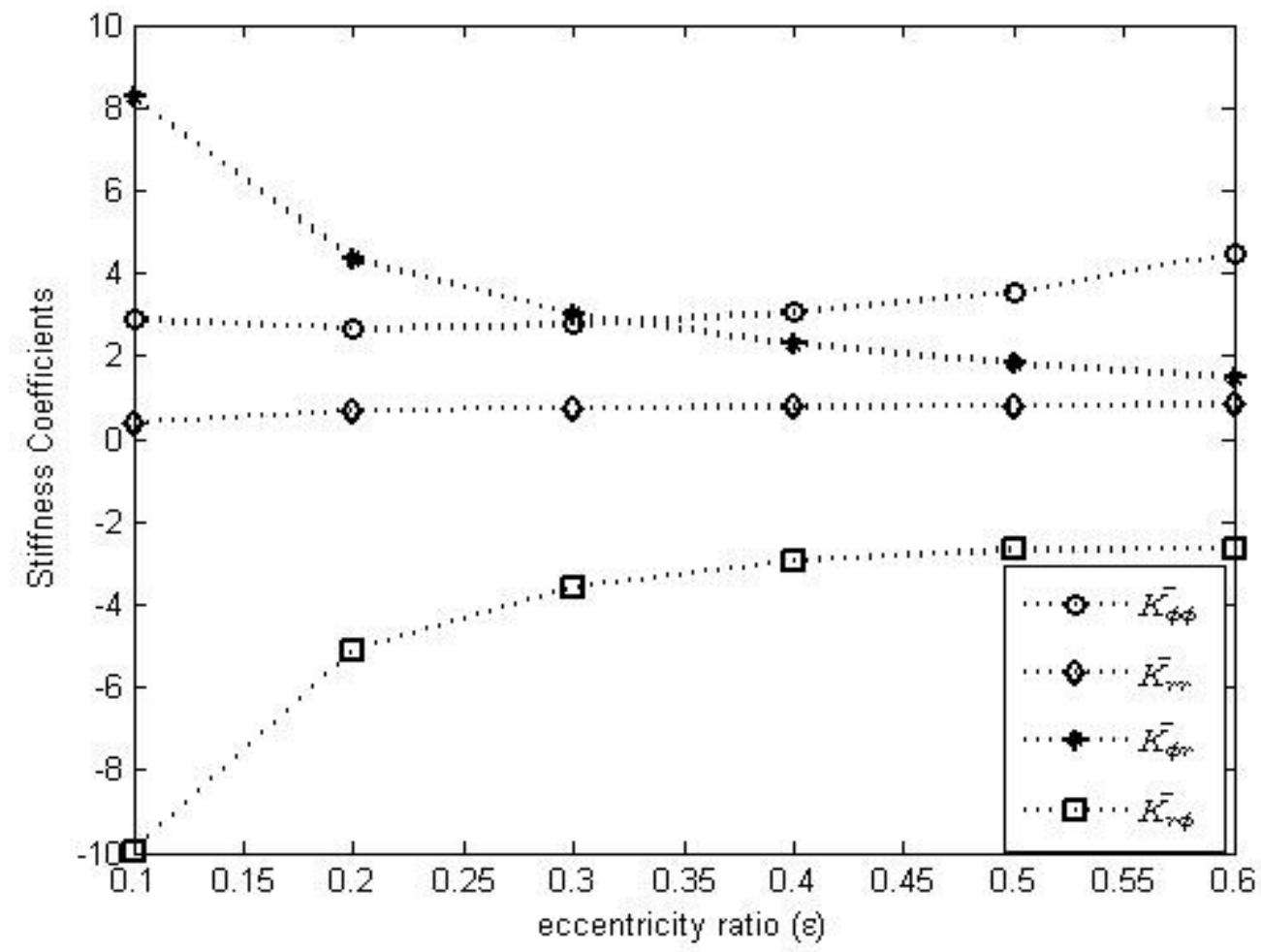

Figure6: Stiffness coefficients vs. Eccentricity $\operatorname{ratio}\left(L / D=1, S_{p}=0.8, \Delta \bar{h}=0.1, \alpha=1, \beta=1\right)$

Figure 7 represents the variation of non-dimensional damping coefficients with eccentricity ratio for $L / D=1, S_{p}=0.8, \Delta \bar{h}=0.1, \alpha=1, \beta=1$. The non-dimensional direct damping coefficients, $\bar{D}_{\phi \phi}$ and $\bar{D}_{r r}$ decrease with increase in eccentricity ratio. The non-dimensional cross damping coefficients, $\bar{D}_{\phi r}$ and $\bar{D}_{r \phi}$ increase slightly with increase in eccentricity ratio. It hasbeen seen from Fig. 8 that the mass parameter increases with increase in eccentricity ratio. Whirl ratio decreases slightly with increase in eccentricity ratio. Textured journal bearing exhibits better stability at higher eccentricity ratios. 
International Journal of Recent advances in Mechanical Engineering (IJMECH) Vol.3, No.3, August 2014

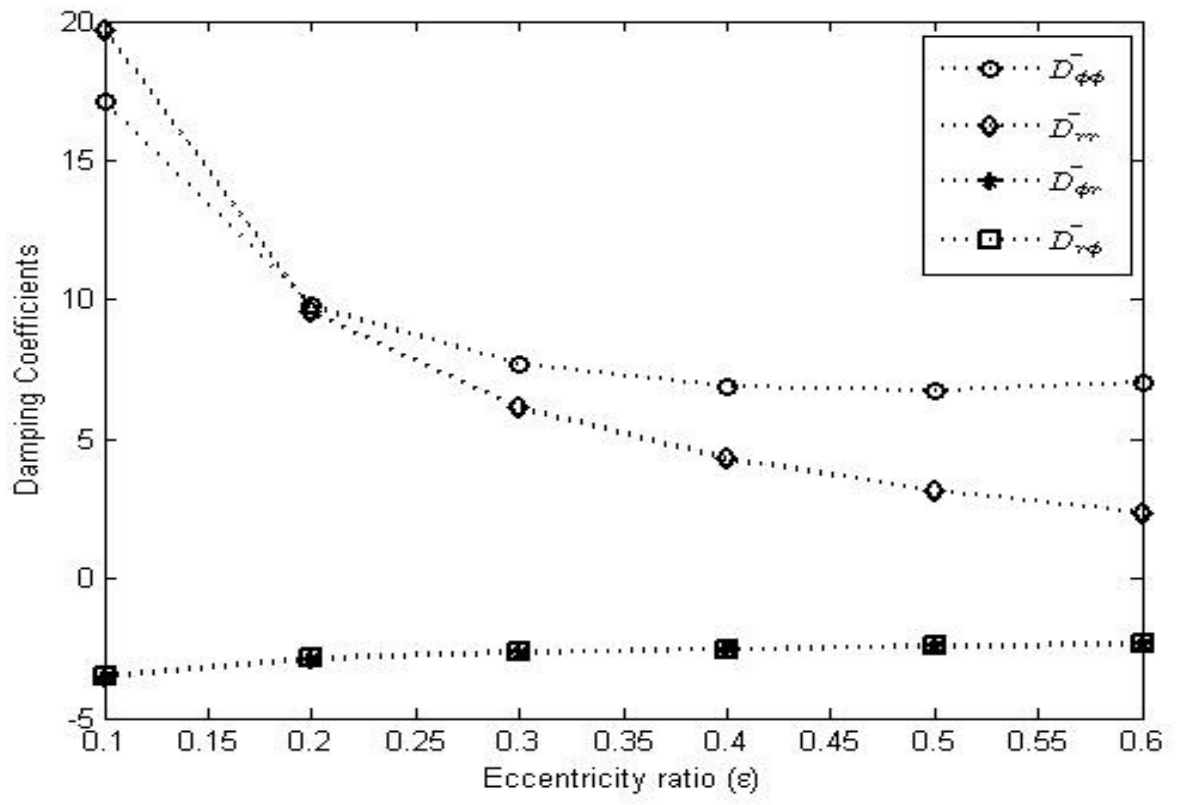

Figure 7:Damping coefficients vs. Eccentricity $\operatorname{ratio}\left(L / D=1, S_{p}=0.8, \Delta \bar{h}=0.1, \alpha=1, \beta=1\right)$

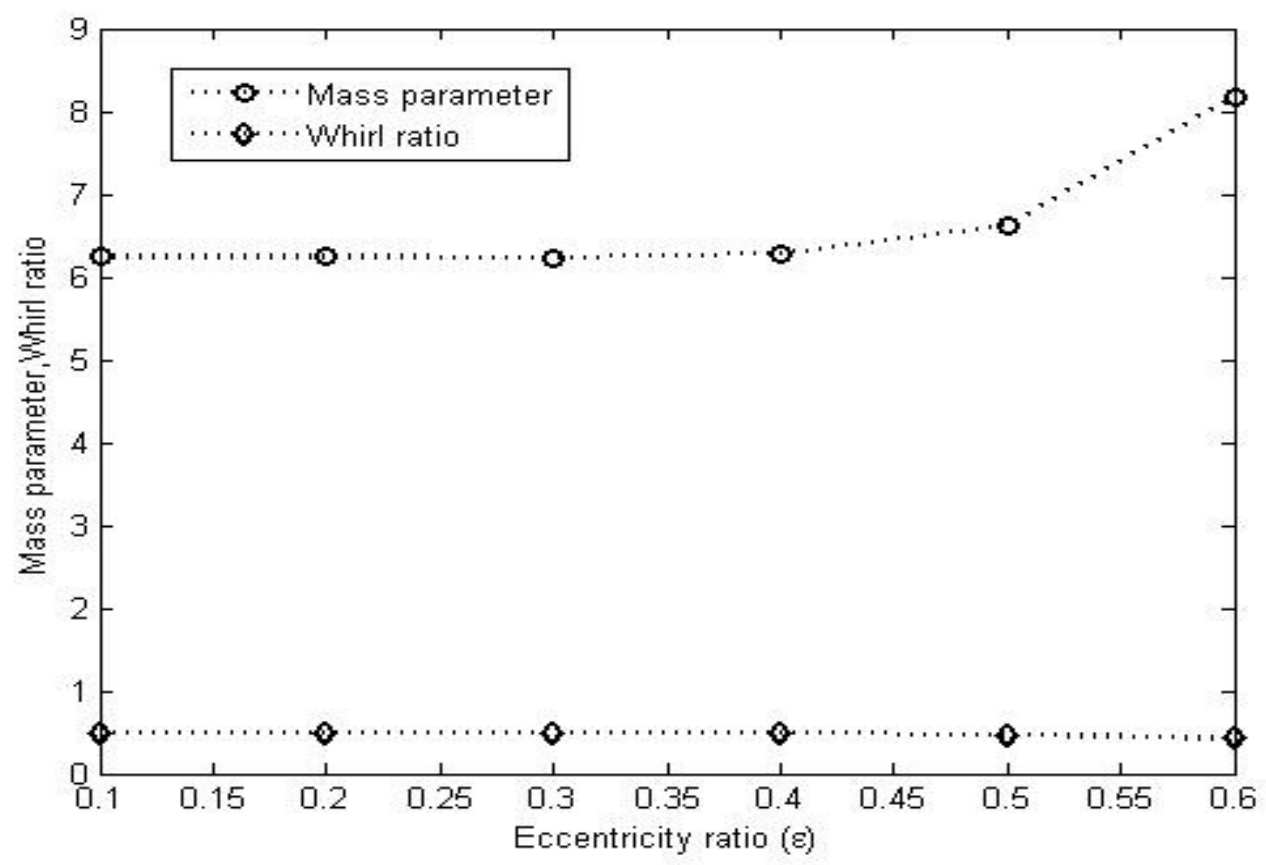

Figure 8: Mass parameter and whirl ratio vs. Eccentricity ratio

$$
\left(L / D=1, S_{p}=0.8, \Delta \bar{h}=0.1, \alpha=1, \beta=1\right)
$$


The effect of texture density on dynamic coefficients and stability has been presented in Figs. 9, 10 and 11 for $L / D=1, \varepsilon=0.6, \Delta \bar{h}=0.1, \alpha=1, \beta=1$. The non-dimensional direct stiffness coefficient, $\bar{K}_{\phi \phi}$ increases with increase in texture density and $\bar{K}_{r r}$ increases first and then decreases with increase in texture density as shown in Fig. 9. The non-dimensional cross stiffness coefficient $\bar{K}_{\phi r}$ decreases firstand then increases slightly with increase in texture density.The cross stiffness coefficient $\bar{K}_{r \phi}$ decreases slightly with texture density. It may be seen from Fig. 10 that the non-dimensional direct damping coefficient, $\bar{D}_{\phi \phi}$ increases with increase in texture density and $\bar{D}_{r r}$, decreases slightly with increase in texture density. The non-dimensional cross stiffness coefficients $\bar{D}_{\phi r}$ and $\bar{D}_{r \phi}$ decrease slightly with increase in texture density.It is observed from Fig. 11 that the mass parameter is the maximum at $20 \%$ texture density.

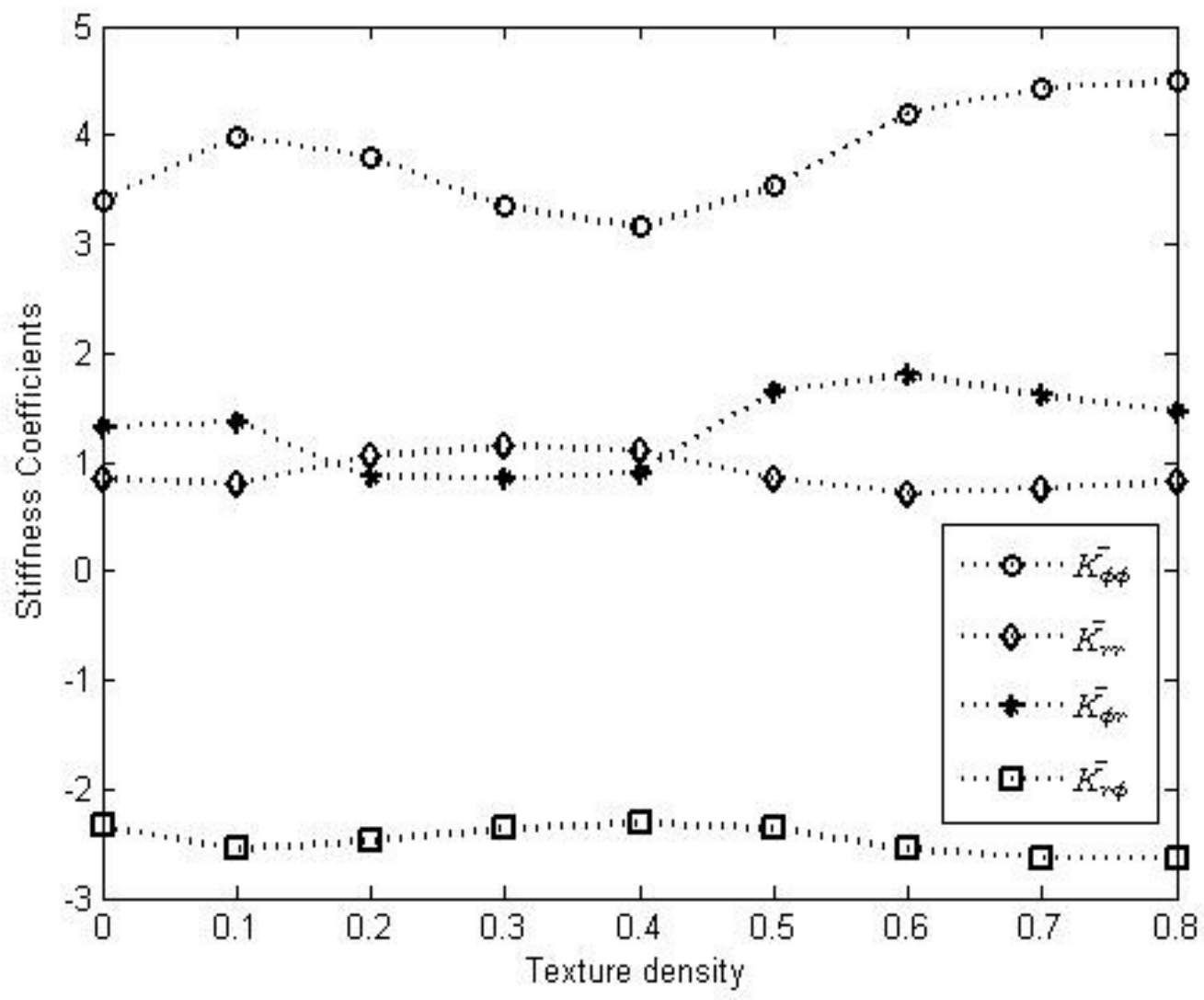

Figure 9: Stiffness coefficients vs. Texture density $(L / D=1, \varepsilon=0.6, \Delta \bar{h}=0.1, \alpha=1, \beta=1)$ 
International Journal of Recent advances in Mechanical Engineering (IJMECH) Vol.3, No.3, August 2014

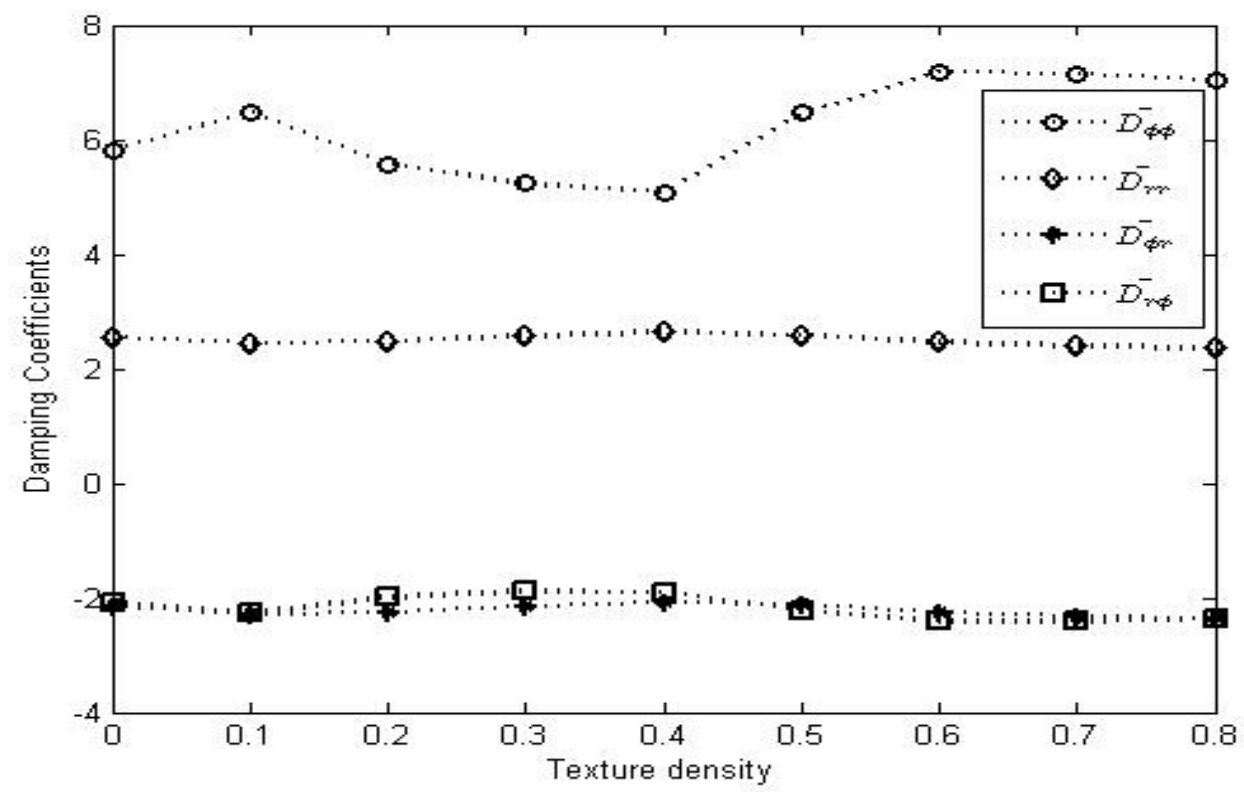

Figure 10:Damping coefficients vs. Texture density $(L / D=1, \varepsilon=0.6, \Delta \bar{h}=0.1, \alpha=1, \beta=1)$

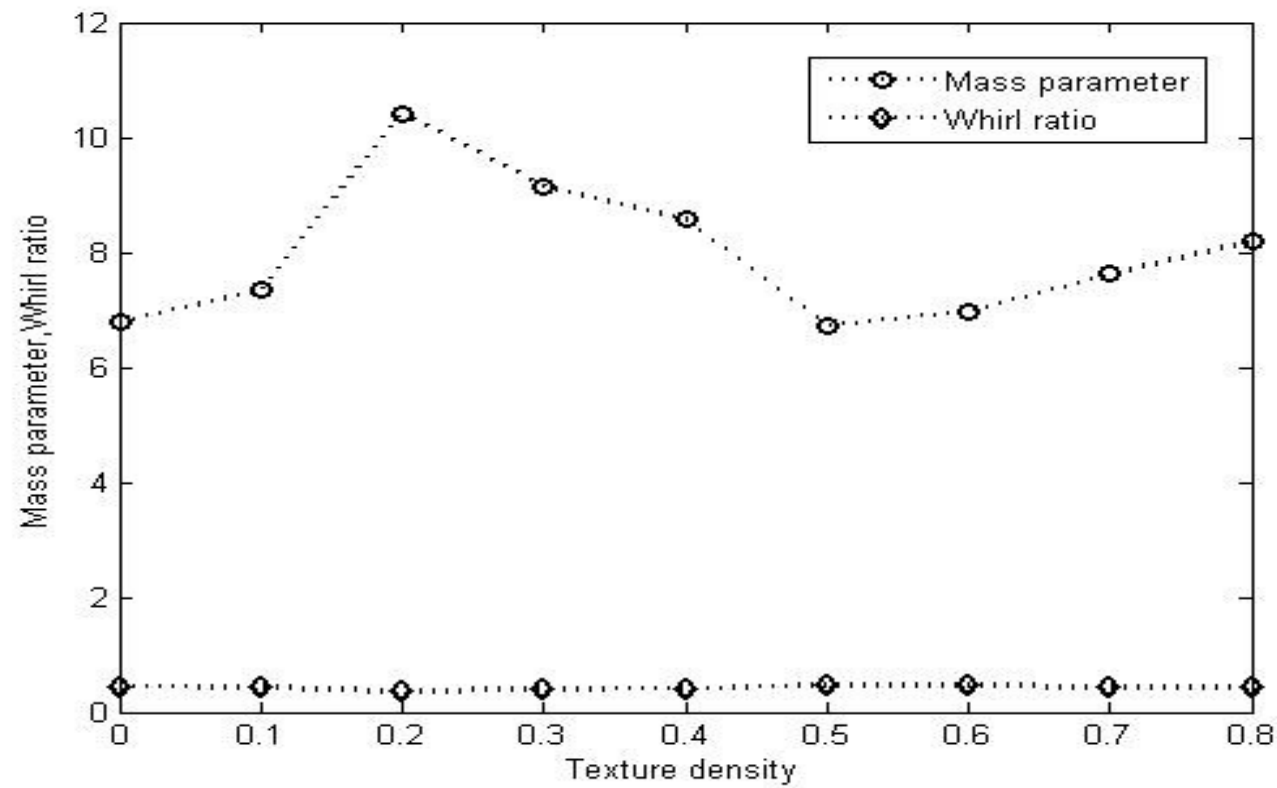

Figure 11: Mass parameter and whirl ratio vs. Texture density( $L / D=1, \varepsilon=0.6, \Delta \bar{h}=0.1, \alpha=1, \beta=1)$ 
International Journal of Recent advances in Mechanical Engineering (IJMECH) Vol.3, No.3, August 2014

\section{Conclusions}

The dynamic coefficients of cylindrical textured journal bearing are presented for increasing texture depth, eccentricity ratio and texture density in the foregoing section. Effect of these parameters on stability has also been presented here. Few important observations are

- Increase in texture depth improves the stability of hydrodynamic journal bearing.

- Stability is a concern for lightly loaded bearing whereas the bearing is textured or untextured. This indicates that texturing does not make lightly loaded bearing highly stable.

- The effect of texture density on stability is very prominent and the maximum stability is obtained at $20 \%$ texture density for the operating conditions presented here. However, optimum texture density at different operating conditions for the maximum stability may be of interest for the bearing designers in future.

\section{REFERENCES}

[1] T. Matsumura, F. Iida, H. Hirosea \& M. Yoshino, (2012) "Micro machining for control of wettability with surface topography”, Journal of Materials Processing Technology, Vol. 212, No. 12, pp26692677.

[2] T. Matsumura, H. Sadakata, H. Makihata \& M. Yoshino, (2013) "Micro fabrication on cylinder surface for control of wettability", Journal of Manufacturing Processes, Vol. 15, No. 1, pp8-13.

[3] N. Tala-Ighil, M. Fillon \& P. Maspeyrot, (2011) "Effect of Textured Area on the Performances of a Hydrodynamic Journal Bearing”,Tribology International, Vol. 44, No. 3, pp211-219.

[4] S. Das, S.K. Guha \& A.K. Chattopadhyay (2004)“Linear stability analysis of hydrodynamic journal bearingsunder micro polar lubrication”, Tribology International,Vol. 38, No. 5, pp500-507.

[5] L. Roy\& S.K.Laha (2009) "Steady state and dynamic characteristics of axial grooved journal bearings", Tribology International, Vol. 42, No. 5, pp754-761.

[6] V. Brizmer \&Y. Kligerman, (2012) “A Laser Surface Textured Journal Bearing”,Journal of Tribology, Vol. 134, No. 3, pp031702-031709.

[7] J. Li\& X. Wang, (2013) "Numerical Simulation of the Influence of the Bulges around Laser Surface Textures on the Tribological Performance",Tribology Transactions, Vol. 56, No. 6, pp1011-1018.

[8] R. Pai, D. SrikanthRao, B. S. Shenoy\& R. S. Pai, (2012) "Stability Characteristics of a Tri-taper Journal Bearing: a Linearized Perturbation Approach", Journal of Materials Research and Technology,Vol. 1, No. 2, pp84-90.

[9] B. Chetti, (2011) "Micro polar Fluids Effects on the Dynamic Characteristics of Four-lobe Journal Bearing", World Academy of Science, Engineering and Technology,Vol. 5, No. 11, pp11-28.

[10] M. Vijaya Kini, R. S. Pai, D. Srikanth Rao, B. S. Shenoy \& R. Pai, (2009) "Effect of Groove Location on the Dynamic Characteristics of Multiple Axial Groove Water Lubricated Journal Bearing", World Academy of Science, Engineering and Technology,Vol. 3, No. 12, pp12-24.

[11] V. Brizmer, Y. Kligerman \& I. Etsion, (2003) "A laser surface textured parallel thrust bearing", Tribology Transactions, Vol. 46, No. 3, pp397-403.

[12] L. Roy \& S. K. Kakoty, (2013) "Optimum Groove Location of Hydrodynamic Journal Bearing Using Genetic Algorithm”, Advances in Tribology, Vol. 2013, Article ID 580367, pp1-13. 